\title{
Kotler, P., Kartajaya, H., Setiawan, I. et Vandercammen, M. (2017). Marketing 4.0 : le passage an digital. Bruxelles, Belgique : De Boeck, 160 p.
}

Myriam Ertz ${ }^{\mathrm{a}}$

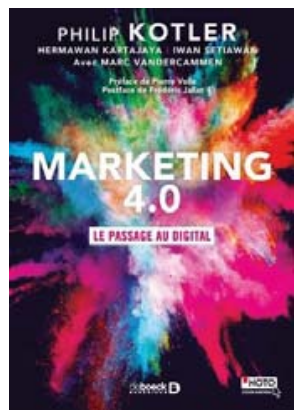

On ne présente plus Philip Kotler, père du marketing moderne, dont les multiples éditions du manuel Marketing Management ont contribué à façonner la pensée et la vision du monde de plusieurs générations de chercheurs et de professionnels du domaine. Après un ouvrage à succès intitulé Marketing 3.0, rédigé en collaboration avec les professionnels indonésiens du marketing Hermawan Katarjaya, fondateur et président-directeur général de MarkPlus inc., et Iwan Setiawan, directeur adjoint de MarkPlus inc. (Kotler, Kartajaya et Setiawan, 2010), Philip Kotler actualise nos connaissances avec la même équipe, cette fois agrémentée de Marc Vandercammen, consultant chevronné en marketing.

Selon Kevin Lane Kaller, professeur de marketing à la Tuck School of Business à Hanover et autre grand théoricien du marketing, personne n'est autant à l'écoute du marketing que Philip Kotler. Ce dernier possèderait une grande capacité à identifier et à interpréter les nouveaux développements et tendances marketing (Kotler et al., 2017). Ainsi, quand il parle, on l'écoute et lorsqu'il écrit, on le lit. Marketing 4.0 a donc été un ouvrage très attendu par les chercheurs et les professionnels du marketing. Alors, que nous disent les auteurs?

\section{Du marketing 1.0 au marketing 4.0}

Le marketing 1.0 représente l'ère du marketing durant laquelle les professionnels du marketing se souciaient principalement de la constitution du meilleur produit au prix le plus bas. Avec le temps, cette vision excessivement centrée sur l'organisation est devenue obsolète, car elle ne permettait plus de réussir dans des marchés de plus en plus concurrentiels et aux consommateurs volatils. Pour s'assurer de l'attention des consommateurs, il fallait donc prendre en compte leurs besoins et leurs désirs afin de concevoir des offres permettant de les satisfaire.

Le marketing 2.0 réfère quant à lui à ce changement de perspective qui implique de placer le client au centre de toutes les attentions des professionnels. Pour réussir, il faut donc avant tout élaborer une stratégie orientée vers les marchés (SOM) (Lachance et al., 2007). Les recherches en marketing durable, en éthique en marketing ou, plus largement, en marketing responsable mirent toutefois en exergue que le développement d'offres marketing répondant à tous les désirs et souhaits des consommateurs pouvait avoir des effets négatifs. Par exemple, le désir de consommer des repas-minute (fast food) ou de fumer du tabac peut avoir des conséquences négatives à moyen ou long terme sur une foule de parties prenantes, dont le consommateur. Dans le même temps, et paradoxalement, pour mieux servir les consommateurs, les interactions avec les marques se réalisèrent de plus en plus par l'intermédiaire de machines, et ces médiations technologiques - à des degrés variables - créèrent une froideur dans les relations commerciales ainsi qu'une déshumanisation des rapports client/marque. La culture potentiellement dommageable dans certains cas du « consommateur-roi » et les innovations technologiques qui ont contribué à le rendre

a Professeure en marketing, responsable du LaboNFC, Université du Québec à Chicoutimi 
plus souverain ont ainsi paradoxalement contribué à une certaine déshumanisation du consommateur ainsi que des rapports entre ce dernier et la marque.

Le marketing 3.0 proposé par Kotler et ses collègues constitue une vision plus « humaine » et moins transactionnelle du marketing. Il vise l'épanouissement de l'esprit humain, plutôt que la satisfaction des moindres caprices et désirs du consommateur. Il encouragerait ainsi les gestionnaires à s'engager dans un marketing axé sur l'humain. Il propose de ne plus uniquement créer de la valeur pour les actionnaires ou les clients, mais également pour les employés et tous les membres de la chaîne de valeur afin d'enclencher une transformation socioculturelle dans laquelle le marketing contribue positivement à la société dans son ensemble. Le marketing 3.0 met aussi l'accent sur la gestion des parties prenantes, sur les entrepreneurs marketing des économies émergentes et sur la durabilité environnementale. Ces préoccupations font écho à des remises en question plus globales, au sein de la communauté marketing, pour plus de responsabilités en marketing.

Le numéro spécial Better marketing for a better world du Journal of Marketing (Moorman, et al., 2018) atteste de ce revirement (ou évolution) du marketing. Avec le marketing 3.0, on note l'introduction d'une certaine charge normative et d'une composante morale dans la consommation et le marketing. Au contraire, avec le marketing 4.0, on se détache légèrement de ce volet moral pour replonger dans les abysses de la technique mercatique.

\section{Les prémisses du marketing 4.0}

Dans les quatre premiers chapitres, les auteurs nous expliquent les changements induits par les nouvelles technologies dans les rapports de pouvoir des consommateurs connectés. Nous sommes passés d'un modèle oligarchique, exclusif et individualiste à un modèle égalitariste, intégré et conformiste. Le client connecté présenterait également des paradoxes puisqu'il oscille entre interactions virtuelles et réelles, et qu'il doit créer un certain équilibre entre les deux sans sombrer excessivement dans l'un ou l'autre pour vivre sainement dans le monde contemporain.

Le client est également de plus en plus averti grâce à l'information massive dont il dispose, mais les sollicitations et notifications diverses le rendent aussi de plus en plus distrait. Enfin, il a un pouvoir énorme qu'il peut utiliser soit pour promouvoir, soit pour dénigrer une marque. Il existerait d'ailleurs des sous-cultures numériques dans ce qu'on appelle plus largement les internautes, dont les jeunes, les femmes et les cybercitoyens (netizens). Ce dernier groupe est défini par Hauben (1992) comme une "population qui, au-delà des frontières, s'activent à développer l'Internet au bénéfice du plus grand nombre » (Kotler et al., 2017, p. 45, trad. libre). Pensons par exemple à certains lanceurs d'alerte comme Chelsea-Bradley Manning en 2010, Edward Snowden en 2013 ou encore Christopher Wylie, l'ex-employé de Cambridge Analytica en 2018, voire Satoshi Nakamoto, l'auteur du livre blanc sur le bitcoin en 2008 (Nakamoto, 2008, cité dans Arsenault et Ertz, 2018). Enfin, l'internaute doit agencer virtuel et réel, concilier apparence et contenu, et réunir humains et technologies.

\section{Les transformations du marketing 4.0}

Le cœur de la contribution et de l'ouvrage arrive en seconde partie, avec les nouvelles perspectives pour le marketing numérique. Les auteurs avancent notamment que le parcours client de l'époque de la préconnectivité est devenu trop simpliste pour le monde numérique. En effet, le modèle conscience appréciation - achat - rachat doit être revu à l'ère de la connectivité. Ainsi, le nouveau modèle se compose plutôt des étapes suivantes : 1) conscience (je connais), 2) intérêt (j'aime), 3) questionnement (je suis convaincu), 4) action (j’achète) et 5) recommandation (je recommande). Cette évolution implique trois grandes mutations : 
1) À l'ère de la préconnectivité, un consommateur détermine sa propre attitude envers une marque. À l'ère de la connectivité, l'attrait de départ est plutôt influencé par la communauté dans laquelle le client évolue pour façonner son choix;

2) À l'ère de la préconnectivité, la fidélité est souvent définie par la fidélisation et le rachat. À l'ère de la connectivité, la fidélité se définit plutôt comme la volonté de défendre une marque : d'en devenir un ambassadeur ou un évangéliste;

3) Pour comprendre les marques, les clients peu connectés participent à des échanges questions-réponses. La tournure que prend l'échange renforce ou amenuise l'attrait initial pour la marque.

L'objectif est donc d'accompagner le consommateur de la prise de conscience à la recommandation à travers ces étapes nouvellement identifiées, et ce, à l'aide de nouveaux points de contact possibles et de nouveaux outils. Parmi les nouveaux points de contact possibles, les publicités en ligne, les médias sociaux, les contacts avec les centres d'appels et de service client ainsi que les infolettres ont une importance singulière.

La bonne nouvelle pour les petites et moyennes organisations ainsi que pour les organisations évoluant dans des territoires éloignés des grands centres urbains, c'est que ces points de contact sont très accessibles, faciles à utiliser et peu coûteux. Ils permettent à ces organisations de mettre en place des activités de marketing concrètes et complètes, puis de redonner ainsi sa place à cette discipline considérée par trop souvent comme trop complexe, coûteuse ou difficile à évaluer. Parmi les outils, on notera l'émergence de nouveaux indicateurs de la productivité marketing, dont les ratios de conversion d'achat (PAR) (acheteurs/notoriété spontanée) et de mobilisation de la marque (recommandation spontanée/notoriété spontanée) (BAR). Là encore, ces outils sont tout indiqués pour les petites structures puisque les données générées en ligne permettent de nourrir ces indicateurs et d'obtenir ainsi des informations précises sur la mesure de la productivité des activités de marketing.

Le modèle, en cinq étapes, présenté ci-dessus permet de mettre en lumière quatre archétypes sectoriels en fonction du nombre de consommateurs se retrouvant à chaque étape. Le modèle traditionnel en marketing, y compris en marketing numérique, est le modèle « entonnoir » puisque de nombreux individus connaissent la marque, mais ils diminuent graduellement en nombre jusqu'à la phase d'achat et de recommandation. Pour 1000 individus ayant conscience de la marque, 100 vont s'y intéresser, 50 vont se renseigner à son sujet (devenant des prospects [leads]), 10 vont acheter et, enfin, 5 vont émettre une recommandation. Mentionnons que cette recommandation peut être positive, négative ou neutre. On a ainsi une déperdition graduelle du contact avec les individus tout au long du parcours client. Le marketing 4.0 vise à remédier à cette situation. On aurait alors trois archétypes supplémentaires :

\section{1) Le modèle poignée de porte}

Il se prête plutôt aux biens de consommation courante. Il représente une situation dans laquelle les consommateurs passent peu de temps à évaluer les options. Puisque les prix sont bas et que les achats sont fréquents et habituels, les efforts de recherche sont inutiles, ce qui écourte la phase de questionnement et augmente la phase d'achat à peu près au même niveau que la phase d'intérêt. La recommandation est ensuite plus faible que l'achat. D'où la forme de poignée de porte. 


\section{2) Le modèle poisson}

Il s'applique à des situations d'achat dans lesquelles les évaluations sont longues et minutieuses, par exemple en commerce inter-organisationnel, mais aussi pour des achats très importants dans le cas des consommateurs. La phase d'intérêt est alors moindre, tandis que la phase de questionnement est beaucoup plus importante.

\section{3) Le modèle trompette}

Il est très fréquent parmi une large gamme d'industries et de produits «style de vie », par exemple les vêtements griffés, les voitures sports ou les montres de luxe. Ce modèle est caractérisé par un niveau d'affinité très élevé. Les consommateurs développent des sentiments forts envers les marques. Ainsi, les phases de conscience, d'intérêt, de questionnement et d'achat suivent le modèle de l'entonnoir, mais la phase de recommandation est beaucoup plus forte, car, même si certains consommateurs ne peuvent pas acheter la marque, ils seront plus que disposés à recommander ces marques à d'autres consommateurs.

Les auteurs suggèrent un cinquième modèle comme modèle idéal à l'ère du numérique.

\section{4) Le modèle noud de papillon}

Il combine les modèles cités précédemment. Dans ce modèle, le motif de nœud papillon reflète les traits essentiels d'une marque parfaite. Chaque consommateur connaissant la marque (connaissance) est prêt à la recommander (recommandation). Si connaissance $=$ recommandation, alors le BAR serait de 1. De plus, puisque l'intérêt pour la marque est tel que chaque consommateur attiré finit par l'acheter, intérêt $=$ achat. On a donc un PAR parfait de 1. Le questionnement représente le centre du nœud et est donc le plus faible puisque tous les consommateurs attirés par la marque ne ressentent pas le besoin de poser des questions. Les auteurs fournissent une cartographie des bonnes pratiques pour se rapprocher de ce modèle idéal.

Le concept d'humanité cher au marketing 3.0 est repris ici puisque les auteurs réitèrent l'importance de construire des marques authentiques et humaines. Cette fois, pourtant, ce sont les nouvelles technologies qui seront mises à contribution avec l'écoute sociale (sur les médias sociaux), la netnographie et la recherche empathique. D'ailleurs, les six attributs des marques centrées sur l'humain et que les marques gagneraient à développer seraient : 1) une apparence agréable, 2) une intelligence hors pair, 3) un caractère social et ouvert aux autres, 4) une sensibilité émouvante, 5) une personnalité forte et 6) une moralité au-dessus de tout soupçon. Pour humaniser leurs relations avec les clients, les marques doivent tout simplement adopter ces qualités humaines afin d'attirer les clients dans une dimension axée sur l'humain. En second lieu, l'aspect authentique se crée de manière privilégiée par le marketing de contenu (en huit étapes) pour éveiller l'intérêt pour la marque. Il convient notamment de raconter des histoires passionnantes sur la marque, un élément rendu possible avec les fonctionnalités de stories sur les médias sociaux, par exemple Instagram. Le contenu devient une technique publicitaire, tandis que le mot-clic devient le slogan.

L'avenir sera également à l'intégration de plus en plus étroite entre les médias numériques et les expériences traditionnelles en magasin. C'est ce qu'on appelle aussi le marketing omnicanal, expliqué en trois étapes. Pour les auteurs, cette tendance augmente en puissance et continuera de le faire. Le commerce mobile jouera un rôle important dans l'économie de l'immédiateté. En magasin, le web-repérage consiste à utiliser les technologies des capteurs (p. ex., la technologie de communication en champ proche [CCP] ou l'identification par radiofréquence) pour interagir en direct avec les consommateurs sur leur mobile en magasin. Lors d'achats virtuels, le furetage devrait être encouragé et l'expérience omnicanale pourra être optimisée par l'analyse de mégadonnées (big data). 
Le concept d'engagement étant central en marketing numérique, les professionnels doivent développer des stratégies de marketing d'engagement pour promouvoir l'affiliation des consommateurs à la marque. Attention, on ne parle pas ici de marketing d'affiliation, mais bien de l'exploitation de la puissance des applications mobiles, de la gestion de la relation client sociale (social CRM) et de la ludification (gamification) pour améliorer l'expérience numérique du client.

Enfin, l'ouvrage insiste sur l'importance de surprendre et d'émerveiller les clients par l'« effet WOW ». Au Québec, ce principe a été popularisé par le professeur Jasmin Bergeron de l'ESG-UQAM. D’ailleurs, "WOW ", c'est également le genre de réaction que pourra avoir le lecteur tout au long de cet ouvrage riche, bien documenté, concret, pratique et constituant un bel approfondissement de l'ouvrage précédent, Marketing 3.0. Avec l'aide de ses collaborateurs, Philip Kotler nous fait encore une fois la démonstration de sa compréhension profonde des enjeux actuels et futurs du marketing.

L’ouvrage est idéal pour les professionnels et les étudiants puisqu'il est émaillé de nombreux exemples, pose des questions de réflexion pertinentes et offre des solutions très concrètes (par étapes) à des enjeux complexes. Il se situe ainsi à mi-chemin entre le manuel et l'essai, ce qui pourra par moments décontenancer certains lecteurs. En effet, peu d'élaborations sont faites sur des concepts théoriques ou des processus complexes, et c'est au lecteur d'approfondir ces connaissances en puisant dans la richesse des sources mentionnées. Cet élagage s'effectue toutefois au bénéfice de l'ouvrage, lequel demeure clair, concis et assez facile à lire. Une recette gagnante pour de nombreuses autres versions à venir, sans doute dans quelques années pour le marketing 5.0!

\section{RÉFÉRENCES}

Arsenault, J. et Ertz, M. (2018). Bitcoin et chaine de blocs : état des lieux et implications pour la gouvernance mondiale. Revue Organisations \& Territoires, 27(2), 117-127. https://doi.org/10.1522/revueot.v27n2.877

Hauben, M. (1992). The Net and netizens: The impact the Net has in people's lives. New York, NY: Columbia University. Repéré à http://www.columbia.edu/ hauben/book-pdf/CHAPTER\%201.pdf

Kotler, P., Kartajaya, H. et Setiawan, I. (2010). Marketing 3.0: From products to customers to the buman spirit. Hoboken, NJ : John Wiley \& Sons.

Lachance, Y., Bousquet, J., Laferté, S. et Marticotte, F. (2007). Marketing stratégique. Québec, QC : Chenelière Éducation.

Moorman, C., Chandy, R., Johar, G. et Roberts, J. (dir.). (2018). Better marketing for a better world [Numéro spécial]. Journal of Marketing. Repéré à https://www.ama.org/2018/11/20/call-for-papers-journal-of-marketing-special-issue-better-marketing-for-abetter-world 